\title{
Chemical Models of Collapsing Envelopes
}

\author{
Edwin A. Bergin \\ Harvard-Smithsonian Center for Astrophysics, Cambridge, MA 02138, \\ $U S A$
}

\begin{abstract}
We discuss recent models of chemical evolution in the developing and collapsing protostellar envelopes associated with low-mass star formation. In particular, the effects of depletion of gas-phase molecules onto grain surfaces is considered. We show that during the middle to late evolutionary stages, prior to the formation of a protostar, various species selectively deplete from the gas phase. The principal pattern of selective depletions is the depletion of sulfur-bearing molecules relative to nitrogen-bearing species: $\mathrm{NH}_{3}$ and $\mathrm{N}_{2} \mathrm{H}^{+}$. This pattern is shown to be insensitive to the details of the dynamics and marginally sensitive to whether the grain mantle is dominated by polar or non-polar molecules. Based on these results we suggest that molecular ions are good tracers of collapsing envelopes. The effects of coupling chemistry and dynamics on the resulting physical evolution are also examined. Particular attention is paid to comparisons between models and observations.
\end{abstract}

\section{Introduction}

The formation of dense molecular condensations, and of stars, involves large changes in the physical properties of initially atomic and eventually molecular gas. These changes have very specific consequences on the chemical interactions of the gas and dust inside the forming cores/stars. In particular, as the density increases molecules in the gas phase will collide with dust grains with greater frequency and, if the molecules stick with any reasonable efficiency, they will deplete from the gas phase. Indeed, molecular depletions have been suggested as the primary chemical indicator of the star formation process (cf. Mundy \& McMullin 1997).

Chemical models of the collapsing envelopes associated with star formation have begun to examine in detail how this process alters the chemistry. These models have focused initially on how to maintain the gas-phase chemistry observed in dense cores, but also on the specific patterns of molecular depletions. This pattern could simply consist of all species depleting on grains at their respective depletion timescales or, with some desorption, removal from the grain surfaces could be selective in nature with some molecules disappearing from the gas phase at early stages while others deplete at later stages. If such a pattern could be isolated, then observations of chemical structure could potentially be used as an indicator of the various phases of the star formation process. 
In this paper we discuss recent models of the "chemistry of star formation". We predominantly focus on chemical models which examine the earliest phases of low mass star formation: collapse of low density molecular envelopes into isolated dense cores and single stars. For a detailed discussion of chemical models for high (and low) mass star formation the reader is referred to the recent review by van Dishoeck \& Blake (1998).

\section{Observations of Molecular Depletions}

The best method to search for molecular depletions is to directly observe molecules in the solid state. This can be done from the ground in many instances (Whittet 1993), but the Infrared Space Observatory has provided the clearest picture to date of the dominant molecules in icy grain mantles (see Ehrenfreund \& Schutte, this volume). However, this method fails if we wish to investigate what happens to the observed gas-phase in molecular clouds as a core condenses. The molecular emission that we observe in star forming regions is from molecules such as CS and $\mathrm{NH}_{3}$, which have abundances as low as $10^{-8}$ relative to $\mathrm{H}_{2}$. Such low abundances, if completely frozen in grain mantles, would produce absorption features too weak to observe. Thus, we are reduced to indirect methods of observing molecules in the gas-phase and searching for emission changes, which could potentially be attributed to depletion. Here, we suffer the additional difficulty of the similar effects on molecular excitation due to changes in abundance or in the physical conditions.

Some careful excitation analyses have been performed and it is certain the molecular depletions have been isolated in several objects. The most striking example is in the starless core L1498 where Kuiper et al. (1996) suggest that CS and $\mathrm{C}_{2} \mathrm{~S}$ are either diminished in abundance or completely absent in the dense central regions, while observations of $\mathrm{NH}_{3}$ show centrally condensed emission. A similar case is in L1544, also a starless core, where $\mathrm{C}_{2} \mathrm{~S}$ emission appears to anti-correlate with both dust continuum emission and $\mathrm{N}_{2} \mathrm{H}^{+}$emission (Ohashi et al. 1999; Ohashi, this volume). An excitation analysis using $\mathrm{N}_{2} \mathrm{H}^{+}$shows that this effect is not due to excitation and is likely the result of $\mathrm{C}_{2} \mathrm{~S}$ depletion in the dense core traced by dust and $\mathrm{N}_{2} \mathrm{H}^{+}$(Plume et al. 2000). Depletion of CO has also been isolated in a cold dense core in IC5146 (Kramer et al. 1999), while similar trends are found in more evolved sources (Blake, van Dishoeck, \& Sargent 1992). For a review of molecular depletions from an observational perspective see Mundy \& McMullin (1997) or Hogerheijde (this volume).

\section{Dynamical Models}

To model the chemical interactions associated with low mass star formation we should look to the current paradigm for the dynamical evolution of collapse or the so-called "standard model of star formation":

1. A magnetically supported core condenses quasistatically via ambipolar diffusion (Basu \& Mouschovias 1994; Lizano \& Shu 1989).

2. During collapse the outer layers approach a $\rho \propto r^{-2}$ density distribution, which is simply hydrostatic equilibrium. 
3. As the density increases in the center, the ionization fraction decreases, and the field lines decouple from the gas.

4. Collapse then ensues in an inside-out fashion (Shu 1977).

An excellent review of our present understanding of star formation can be found in Hartmann (1998). For critical discussions of the standard model see Whitworth et al. (1994) and Nakano (1998).

\section{Gas-Grain Interactions}

An important part of any coupled chemical and dynamical models is the physics of the interaction between molecules and grain surfaces. Two of the most relevant processes are the sticking coefficient and the binding energy. (1) The sticking coefficient is simply how often a molecule will stick to the surface after a collision with a grain and is typically assumed to be between 0.1 and 1 . The theory behind this assumption is discussed by Williams (1993). (2) The process by which molecules are adsorbed or bound onto grain surfaces is known as physical adsorption. When a molecule approaches a grain there is an induced dipole-dipole (van der Waals) interaction between the gas phase molecule and whatever species is dominating the grain surface, either an exposed silicate surface, or more likely $\mathrm{H}_{2} \mathrm{O}$ or $\mathrm{CO}$ molecules. This interaction results in an attractive force which binds the molecule to the surface in a potential well (e.g. Tielens \& Allamandola 1987). Because this is an induced dipole-dipole interaction the strength of the attraction depends on the polarizability of the molecules involved, and a polar molecule, such as $\mathrm{H}_{2} \mathrm{O}$, presents a stronger binding surface than a surface covered by a non-polar molecule, such as CO.

\section{Chemical Models}

The large scale of the star formation problem, which requires coupling very complex hydrodynamical codes with complicated chemical models, renders a complete examination difficult. The models relevant for this discussion are those that have extracted parameterized fits to the density evolution from the dynamical simulations and used these in concert with complex chemical codes. Of these models, each incorporates gas-grain interactions, but they vary greatly in the desorption mechanism theorized to remove molecules from the grain surface. The models are ordered below in terms of increasing desorption efficiency.

Rawlings et al. (1992) examine the inside-out collapse solution of Shu (1977) with no desorption. Bergin \& Langer (1997) use the ambipolar diffusion density evolution given in Basu \& Mouschovias (1994), along with another dynamical solution. These models include cosmic-ray spot heating wherein a cosmic ray strikes a grain and evaporates off molecules from a localized hot spot near the impact. Willacy, Rawlings, \& Williams (1994) use the Shu solution and incorporate both cosmic-ray heating and heating due to $\mathrm{H}_{2}$ formation. Shalabiea \& Greenberg (1997) also use the Shu solution with efficient desorption via grain explosions. One common assumption is that at the start of the evolution all of the hydrogen is in molecular form. 


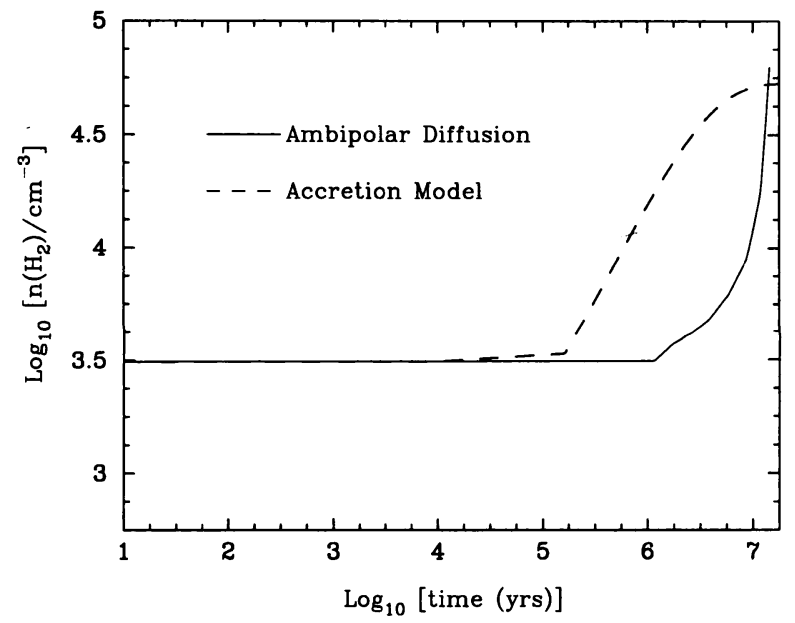

Figure 1. The evolution of the density of molecular hydrogen as a function of time for the evolution through ambipolar diffusion of Basu $\&$ Mouschovias (1994) (solid line) and an accretion model (dashed line).

The above models each assume isothermal evolution at $10 \mathrm{~K}$ and, hence, are appropriate for the initial stages of collapse or for the outer envelopes once a protostar has formed in the innermost regions. Ceccarelli et al. (1996) include the effects of central heating due to the protostar and these results are discussed in $\S 6$. Below we will briefly discuss the models of Rawlings et al. (1992) and, in greater detail, those of Bergin \& Langer (1997).

\subsection{Rawlings et al. 1992}

These models, using the Shu (1977) inside-out collapse solution, examine the evolution at every point in the collapsing envelope at all times, but include depletion with no desorption. Therefore, all molecules deplete from the gas phase as the density increases. One interesting result of these simulations is that when the neutral species deplete the abundances of molecular ions increases. This is the result of a constant ion formation rate from cosmic rays, coupled with a decreasing ion destruction rate from reactions with the depleted neutral species. Eventually, the molecular ions will disappear from the gas phase as their parent molecules deplete (e.g. $\mathrm{CO}$ for $\mathrm{HCO}^{+}$or $\mathrm{N}_{2}$ for $\mathrm{N}_{2} \mathrm{H}^{+}$). This suggests that molecular ions should be good tracers of collapsing envelopes.

\subsection{Bergin \& Langer 1997}

This work investigates the evolution of a single parcel of gas deep inside a core using two dynamical solutions. The first is based on the work of Basu \& Mouschovias (1994) who examined the formation of a dense cloud core via diffusion of magnetic flux. The second is a phenomenological model wherein a static dense core is accreting material from a low density halo and the gas is brought from low to high density within a few free-fall times. The time evolution of density for these models is shown in Figure 1. The chemical models included 

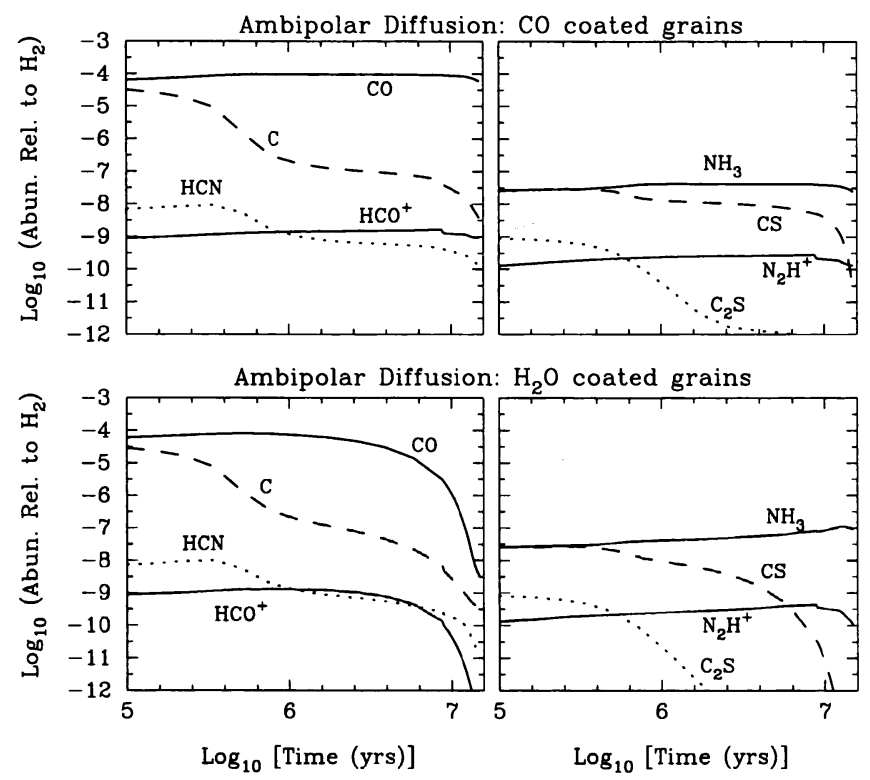

Figure 2. Chemical abundances relative to $\mathrm{H}_{2}$ as a function of time for the ambipolar diffusion model. The top panel shows the chemical evolution for a model with CO-coated grains and the bottom for $\mathrm{H}_{2} \mathrm{O}$ -coated grains.

cosmic-ray spot heating, and examined solutions where grains are coated with a layer of water molecules (strongly binding) and, alternately, a layer of CO molecules (weakly binding).

For the chemical models at $t=0$, carbon and metal atoms are in ionized form, while the oxygen and nitrogen atoms are neutral. All species are depleted from solar abundances, with the heavy metals more severely depleted. A dense pre-protostellar core forms from moderately dense gas and dust composed of a mix of atomic and molecular species. To establish this state the chemistry is allowed to evolve at constant density for $10^{5}$ yrs and these abundances are used as initial conditions for the time-dependent density model.

Chemical abundances as a function of time for the first model of dynamical evolution via diffusion of magnetic flux are shown in Figure 2. In the model with CO-coated grains, abundances exhibit only small changes until $t \sim 3 \times 10^{5}$ yrs. At this time the abundances of simple and complex carbon-bearing species begin to decline rapidly. This change is not the result of any density increase (see Figure 1). Rather the rapid decline in the abundances of $\mathrm{HCN}$ and $\mathrm{C}_{2} \mathrm{~S}$ is related to the sharp drop in the abundance of neutral carbon resulting from the onset of $\mathrm{CO}$ formation. There is also no discernible effect of molecular depletion until $t \sim 10^{7}$ yr $\left(n_{\mathrm{H}_{2}}>10^{4} \mathrm{~cm}^{-3}\right)$ when the abundances of CS and $\mathrm{C}_{2} \mathrm{~S}$ drop precipitously as these molecules deplete onto grain surfaces. Even at late evolutionary stages a reasonable amount of $\mathrm{CO}, \mathrm{HCO}^{+}, \mathrm{HCN}, \mathrm{NH}_{3}$, and $\mathrm{N}_{2} \mathrm{H}^{+}$ remain in the gas. 


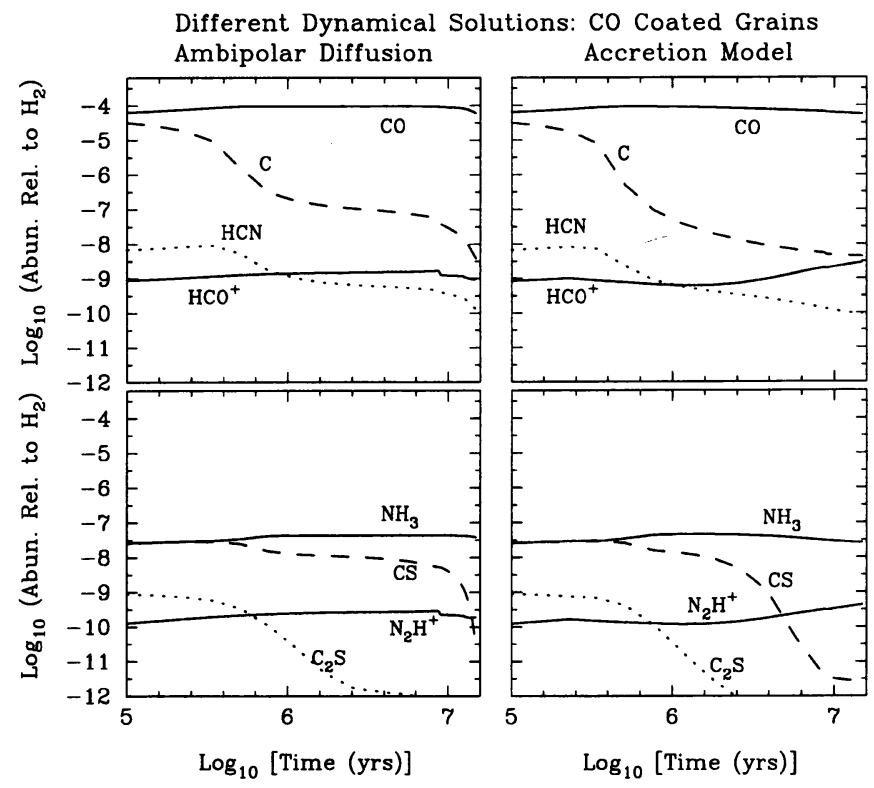

Figure 3. The chemical abundances relative to $\mathrm{H}_{2}$ as a function of time comparing the two dynamical solutions: ambipolar diffusion model (left-hand panels), accretion model (right-hand panels). These models are for CO-coated grains.

This depletion pattern is reminiscent of the chemical structure observed in L1498, in L1544, and in other cores (see $§ 3$; Ohashi, this volume) and is simply understood by the way the various atomic pools interact with the grains. For the $\mathrm{C}, \mathrm{O}$, and $\mathrm{N}$ pools the dominant species are highly non-polar $\left(\mathrm{CO}, \mathrm{O}, \mathrm{O}_{2}\right.$, $\mathrm{N}, \mathrm{N}_{2}$ ) and, while these species are depleting onto grains, cosmic-ray desorption is able to remove them from the weakly binding $\mathrm{CO}$ surface. These molecules are processed in the gas phase by cosmic-ray induced photons and reactions with $\mathrm{He}^{+}$atoms, eventually creating complex polar molecules that will deplete such as $\mathrm{HCN}$ or $\mathrm{H}_{2} \mathrm{CO}$. However, the dominant species (e.g. $\mathrm{CO}, \mathrm{N}_{2}$ ) are more abundant (by three or four orders of magnitude) than more complex molecules and essentially carry the chemistry on their backs. The opposite is true for the sulfur chemistry, the dominant molecules are CS and SO, which are polar molecules with high binding potentials (even on a CO surface). These species deplete onto grain surfaces and are very difficult to remove. Hence, the sulfur chemistry is halted while the $\mathrm{C}, \mathrm{O}$, and $\mathrm{N}$ chemistry continues until densities reach levels that depletion overwhelms desorption.

Figure 2 also shows the evolution of the ambipolar diffusion model for the case where the grain mantles are dominated by water ice. Here the binding energies are much higher than in the previous case and most molecules disappear from the gas phase, except for $\mathrm{N}_{2} \mathrm{H}^{+}$and $\mathrm{NH}_{3}$. This result is interesting and is due to the fact that $\mathrm{N}_{2}$, the parent molecule of both $\mathrm{N}_{2} \mathrm{H}^{+}$and $\mathrm{NH}_{3}$, is one of the most volatile molecules in star forming clouds. Thus, $\mathrm{N}_{2} \mathrm{H}^{+}$and $\mathrm{NH}_{3}$ are suggested by these models to be the best tracers of dense collapsing gas. 


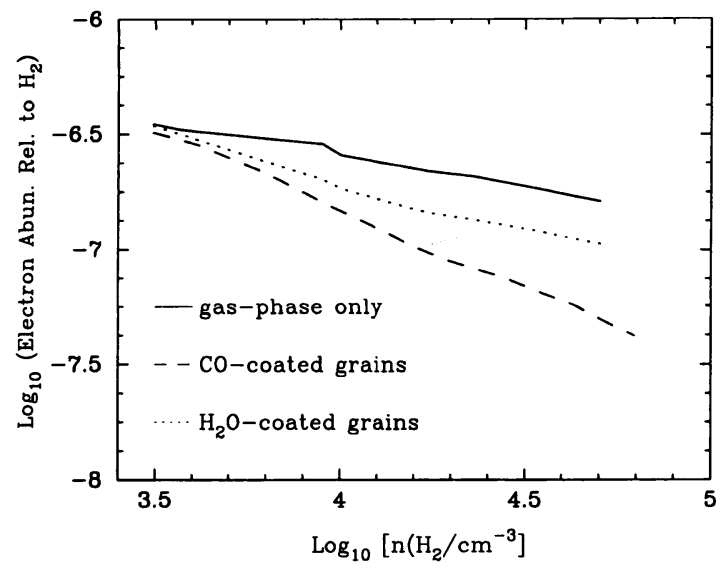

Figure 4. The electron abundance relative to $\mathrm{H}_{2}$ as a function of density for three different chemistry models.

The above models examined the chemistry using the dynamical solutions appropriate for core formation via the diffusion of magnetic flux. In Figure 3 we examine the effect of changing the dynamical solution. In the figure we show the previous model of ambipolar diffusion side-by-side with the accretion model discussed earlier. We only present results for the model with CO-coated grains, but similar effects are found for water ice dominated mantles (see Bergin \& Langer 1997). We find little change in the carbon chemistry between the solutions (top panels). For the sulfur and nitrogen chemistry, presented in the bottom panels, there are some differences, mainly that CS begins to deplete from the gas phase at earlier times in the accretion model. The explanation for this difference can be found in Figure 1 where we see that the gas spends a larger amount of time in a high density state in the accretion model than for ambipolar diffusion, which results in a shorter depletion timescale for CS.

In general, we conclude that changing the dynamical model has little effect on the solution. This result is quite robust in the sense that similar results would be found for any dynamical model that increases with time or is centrally concentrated. Therefore, the density gradient that results from core condensation is accompanied by chemical gradients, with the inner parts of the core representing high density chemistry (with significant CS and CCS depletions onto grains and $\mathrm{N}_{2} \mathrm{H}^{+}$remaining in the gas phase) and the outer parts representing the original low density molecular composition. The size scale of the CS or CCS "hole" will primarily depend on the radius where the gas density is $Z 2 \times 10^{4}$ $\mathrm{cm}^{-3}$, which is roughly the density at which the sulfur molecules deplete. Using the density profiles of B335 from Zhou et al. (1993) or L1689B from André, Ward-Thompson, \& Motte (1996), we estimate that sulfur depletion occurs at radii $<0.025 \mathrm{pc}$ (or $\sim 30-40^{\prime \prime}$ at the distance of Taurus). This is comparable to the size of the CCS depletion zone observed in L1498 and L1544 (Kuiper et al. 1996; Ohashi et al. 1999). More efficient desorption might change these results, but observations of molecular depletions are beginning to rule out a very efficient mechanism. However, these results would suggest that chemistry cannot be used 
Table 1. Chemical evolution and star formation.

\begin{tabular}{|c|c|c|c|}
\hline Evolutionary Phase & Molecular Abundances & $n_{\mathrm{H}_{2}}\left(\mathrm{~cm}^{-3}\right)$ & Example \\
\hline Dense Core Formation & Normal Abundances & $\sim 3 \times 10^{3}$ & HCL2 \\
\hline $\begin{array}{l}\text { Evolving Cores: } \\
\text { (Early Stages) } \\
\text { (Late Stages) }\end{array}$ & $\begin{array}{l}\text { Little } \mathrm{CS} \text { and } \mathrm{C}_{2} \mathrm{~S} \text { depletion } \\
\text { Increasing } \mathrm{CS} \text { and } \mathrm{C}_{2} \mathrm{~S} \text { depletion } \\
\mathrm{N}_{2} \mathrm{H}^{+} \text {and } \mathrm{HCO}^{+} \text {tracing envelope }\end{array}$ & $\begin{array}{l}\sim 10^{4} \\
\sim 3 \times 10^{4}\end{array}$ & $\begin{array}{l}\text { TMC-1 } \\
\text { L1498 }\end{array}$ \\
\hline Collapsing Phase & $\begin{array}{l}\text { Significant } \mathrm{CS} \text { and } \mathrm{C}_{2} \mathrm{~S} \text { depletion } \\
\mathrm{HCO}^{+} \text {depleting prior to } \mathrm{N}_{2} \mathrm{H}^{+}\end{array}$ & $>10^{5}$ & (?) \\
\hline $\begin{array}{l}\text { Protostar Formation } \\
\text { (outer envelope) }\end{array}$ & $\begin{array}{l}\text { Significant depletion, strongly } \\
\text { dependent on mantle properties }\end{array}$ & $>10^{6}$ & $\begin{array}{l}\text { HL Tau, } \\
\text { DG Tau }\end{array}$ \\
\hline $\begin{array}{l}\text { (inner envelope) } \\
\text { stellar heating }\end{array}$ & $\begin{array}{l}\text { Release of mantle species, } \\
\text { high- } \mathrm{T} \text { chemistry }\left(\mathrm{H}_{2} \mathrm{O}\right) \\
\text { depression of } \mathrm{HCO}^{+} \text {and } \mathrm{N}_{2} \mathrm{H}^{+} \\
\text {abundances at center }\end{array}$ & $>10^{7}$ & IRAS16293 \\
\hline Onset of Wind Activity & $\begin{array}{l}\text { Full liberation of grain mantles near } \\
\text { star, shocked regions }\left(\mathrm{H}_{2} \mathrm{O} \text { and } \mathrm{SiO}\right)\end{array}$ & & Orion IRc2 \\
\hline Late Embedded Period & Normal Abundances & & \\
\hline
\end{tabular}

to help constrain the dynamics and that the assignment of a true "chemical" timescale awaits a better understanding of the dynamics.

\section{Chemistry and Star Formation}

It appears clear from the preceding discussion that the process of star formation does have significant and potentially observable effects on the chemistry of the gas. However, chemistry is also an important part of the dynamical evolution. As an example we show in Figure 4 the ionization fraction as a function of density taken from Bergin \& Langer (1997), using the ambipolar diffusion dynamical solutions of Basu \& Mouschovias (1994). A pure gas-phase model is shown, in addition to gas-grain simulations using $\mathrm{CO}$ and $\mathrm{H}_{2} \mathrm{O}$ mantles. Clearly the electron abundance is a function of density; however, what is new in these results is that the character of the density dependence changes with the strength of the gas-grain interaction. In this instance, with the density evolution taken from a dynamical model driven by ion-neutral drift, such differences could be important, which is an excellent argument for the incorporation of chemical interactions into dynamical models.

In summary, over the past several years the wealth of observational data and theoretical chemical simulations has provided our field with important clues to the puzzle of star formation. At the previous IAU conference Mundy \& McMullin (1997) provided a general outline of the chemical evolutionary sequence associated with each phase of the star formation process. We believe that the observations and theory are converging to a point that allows for greater specification. We provide a more detailed evolutionary sequence in Table 1 . The dynamical evolution begins with a core at low density with normal abundances. As the core evolves the principal chemical signature of condensation is the de- 
pletion of sulfur-bearing molecules relative to the nitrogen-bearing species, $\mathrm{NH}_{3}$ and $\mathrm{N}_{2} \mathrm{H}^{+}$. Thus, for early stages, prior to collapse CS or $\mathrm{C}_{2} \mathrm{~S}$ are good probes; during collapse the best tracers of the cold dense envelope are the molecular ions: $\mathrm{N}_{2} \mathrm{H}^{+}$and $\mathrm{HCO}^{+}$. At later evolutionary stages $\mathrm{HCO}^{+}$should deplete prior to $\mathrm{N}_{2} \mathrm{H}^{+}$, making $\mathrm{N}_{2} \mathrm{H}^{+}$the most robust tracer of dense gas. The above conclusion is not applicable for all stages, once a star forms in the center it will locally heat the surrounding material releasing mantle species and, perhaps, allowing for high temperature chemistry to create water (Ceccarelli et al. 1996). The flood of neutrals into the gas phase will destroy molecular ions, making neutral molecules the better tracers for the inner envelope. At the final stages, developing winds will interact with surrounding gas producing shocked regions which can be probed by $\mathrm{SiO}$ and $\mathrm{H}_{2} \mathrm{O}$.

Acknowledgments. The author is immensely grateful for the help, advice, and fruitful collaboration with W. Langer, and to D. Wilner for a careful reading of the manuscript.

\section{References}

André, P., Ward-Thompson, D., \& Motte, F. 1996, A\&A, 314, 625

Basu, S. \& Mouschovias, T.Ch. 1994, ApJ, 432, 720

Bergin, E.A. \& Langer, W.D. 1997, ApJ, 486, 316

Blake, G.A., van Dishoeck, E.F., \& Sargent, A.I. 1992, ApJ, 391, L99

Ceccarelli, C., Hollenbach, D.J., \& Tielens, A.G.G.M. 1996, ApJ, 471, 400

Hartmann, L. 1998, Accretion Processes in Star Formation, Cambridge Univ. Press: Cambridge

Kramer, C., Alves, J., Lada, C.J., Lada, E.A., Sievers, A., Ungerechts, H., \& Walmsley, C.M. 1999, A\&A, 342, 257

Kuiper, T.B.H., Langer, W.D., \& Velusamy, T. 1996, ApJ, 468, 761

Lizano, S. \& Shu, F. II 1989, ApJ, 342, 834

Mundy, L.G. \& McMullin, J.P. 1997, in Molecules in Astrophysics: Probes and

Processes, ed. E.F. van Dishoeck, Kluwer: Dordrecht, 183

Nakano, T. 1998, ApJ, 494, 587

Ohashi, N., Lee, S.W., Wilner, D.J., \& Hayashi, M. 1999, ApJ, 518, L41

Plume, R., Bergin E.A, Caselli, P., \& Myers, P.C. 2000, in prepartion

Rawlings, J.M.C., Hartquist, Menten, K.M., \& Williams, D.A. 1992, MNRAS, 255,471

Shalabiea, O.M. \& Greenberg, J.M. 1995, A\&A, 303, 233

Shu, F. II 1977, ApJ, 214, 488

Tielens, A.G.G.M. \& Allamandola, L.J. 1987, in Interstellar Processes, eds. D.J. Hollenbach \& H.A. Thronson, Reidel:Dordrecht, 397

Whittet, D.C.B. 1993, in Dust and Chemistry in Astronomy, eds. T.J. Millar \& D.A. Williams, Institute of Physics Publishing:Bristol, 9

Whitworth, A.P., Bhattal, A.S., Francis, N., \& Watkins, S.J. 1996, MNRAS, 283,1061

Willacy, K., Rawlings, J.M.C., \& Williams, D.A. 1994, MNRAS, 269, 921

Williams, D.A. 1993, in Dust and Chemistry in Astronomy, eds. T.J. Millar \&

D.A. Williams, Institute of Physics Publishing:Bristol, 143

van Dishoeck, E.F. \& Blake, G.A. 1998, ARA\&A, 36, 317

Zhou, S., Evans, N.J., Koempe, C., \& Walmsley, C.M. 1993, ApJ, 404, 232 


\section{Discussion}

E. van Dishoeck: Can you comment on the sensitivity of your results for the evolving cores to the efficiency of desorption? If the depletions in the center of the cloud are indeed so high as observations suggest ( $>$ factor 10), we may be observing primarily the skin of the cores. Can you comment on the importance of UV radiation in that region?

E. Bergin: The S-bearing molecular are highly bound to the grain surface and only the most efficient mechanism such as, perhaps, chemical explosions could change the results. However a very efficient desorption mechanism is ruled out by observations. The UV field is indeed a good way to remove molecules from grains at cloud edges. However the UV field also tends to destroy the molecules that desorb.

M. Walmsley: What form is most gas phase sulfur in your models? Is a large fraction of gas phase $\mathrm{S}$ in dense clouds in atomic form?

E. Bergin: The models do predict a large abundance of atomic sulfur. However the initial depletion times are long enough to form both CS and SO, prior to depletion. At higher densities atomic $\mathrm{S}$ and the S-bearing molecules deplete onto grains stopping the sulfur chemistry.

J. Rawlings: We have observed CS toward the central region of B335 and find that it accounts for nearly all of the sulfur budget - with no evidence for depletion. The CS line profiles show good evidence for infall and so appear to be associated with the collapsing cloud. Would you like to comment on this?

E. Bergin: Often high resolution images taken from $\mathrm{mm}$ interferometers show evidence for different structure than seen in single dish measurements.

D. Williams: Some species are known to be very little depleted in diffuse clouds, but heavily depleted in dark clouds. How do you treat such species in your models?

E. Bergin: These models start with heavy metals depleted from diffuse cloud values, while carbon, oxygen, and nitrogen start with diffuse cloud values.

$P$. Caselli: There are many uncertainties involving surface chemistry. You showed that different binding energies of molecular species onto dust grains give significantly different gas phase chemical compositions. Could it be possible to carry out observations of "crucial" molecular species (the ones showing the highest sensitivity to binding energy variations) toward quiescent cloud cores to actually determine the more appropriate value of binding energy and then reduce the number of free parameters in chemical models? Which species should be used for this purpose?

E. Bergin: The models do predict that little $\mathrm{CO}$ and $\mathrm{HCO}^{+}$deplete onto grains at $n\left(\mathrm{H}_{2}\right)<10^{5} \mathrm{~cm}^{-3}$ for $\mathrm{CO}$-coated grains. For water ice-coated grains both $\mathrm{CO}$ and $\mathrm{HCO}^{+}$will deplete. However the actual mantles in clouds might at times be either CO- or water-coated, or even partially both. So, it is unclear whether these molecules can be used as probes of the binding energy. 